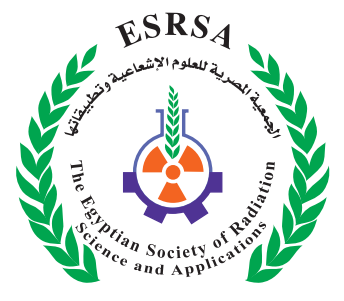

J. Nucl. Tech. Appl. Sci., Vol. 8, PP. 125 : 134 (2020)

\title{
Role of 5-Fluorouracil Chitosan Nanoparticles on Decreasing the Oxidative Stress in Mice Bearing Ehrlich Carcinoma
}

\author{
Huda, S.M. ${ }^{1}$; Neamat, H.A. ${ }^{2}$; Nefisa, H.M. ${ }^{1}$ and Usama, Z S. ${ }^{2}$
}

Received: 21/07/2020

Accepted: 17/09/2020

DOI: $10.21608 /$ jntas.2020.36757.1025

E.mail:nhanafi58@yahoo.com

\section{KEYWORDS}

5-fluorouracil chitosan nanoparticles (5FUCNPs), Ehrlich carcinoma, angiogenic markers.

\section{ABSTRACT}

5-Fluorouracil (5-FU) as anticancer drug has many side effects. Site-specific delivery of 5-FU would reduce the systemic side effects and provide effective and safe therapy. Chitosan nanoparticles (CNPs) are used in drug delivery systems. In the present study the effects of 5-fluorouracil chitosan nanoparticles (5FUCNPs) on decreasing the oxidative stress were investigating within a model of female mice bearing solid Ehrlich carcinoma (EC). 7 days After solid tumor induction, 5-FUCNPs were administrated by gavages $(0.5 \mathrm{mg} / \mathrm{kg}$ body weight $)$ to mice for 15 days. Tumor size was monitored; oxidative stress markers were assessed. In addition, the angiogenic markers concentrations were evaluated. In vitro, 5FUCNPs showed high cytotoxic effect on EC tumor cells. Gavages of EC-bearing mice with 5FUCNPs significantly reduced tumor size, increased MDA level, decreased GSH level and recorded great destruction in tumor tissues. Meanwhile, in liver tissue MDA level significantly decreased and GSH level increased. On the other hand, a significant decrease in the levels of angiogenic markers were recorded. In conclusion: CNPs as a drug carrier for 5-FU have a role in reducing tumor growth and may represent a novel class of anticancer drug.

1. Zoology Department, Faculty of Science, Ain Shams University.

2. Radiation biology Department, National Canter for Radiation, Research and Technology, Egyptian Atomic Energy Authority. 


\section{INTRODUCTION}

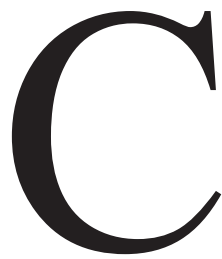

ancer, known medically as a malignant neoplasm, is a broad group of various diseases, all involving unregulated cell growth (Estanqueiro et al., 2015).

Experimental tumors have great importance in modelling of carcinogenesis studies. Ehrlich tumor is a transplantable neoplasia from a malign epithelium, which corresponds to mammary adenocarcinoma in female mice (Ozaslan et al., 2011).

Nanoparticles have been shown to be delivered to specific sites by size-dependent passive targeting (Alvarez-Lorenzo et al., 2011). Chitosan (Cs) is considered one of the most valuable polymer for biomedical and pharmaceutical applications due to its biodegradability, biocompatibility, antimicrobial, non-toxicity, and anti-tumor properties. Nanoparticles, microspheres, hydrogels, films, and fibers are typical chitosan based forms for biomedical and pharmaceutical applications such as transdermal drug delivery (Ravi Kumar, 2000). 5-Fluorouracil (5-FU) is a cytotoxic drug, which interferes with nucleic acid synthesis, inhibits DNA synthesis, and eventually halts cell growth (Burns and Beland, 1983). It is extensively used to treat solid tumors such as liver, breast, colorectal and brain cancer. However, 5-FU is rapidly metabolized, associated with wide ranging side effects among which severe gastrointestinal toxicity, hematologic disturbance, and severe bone marrow deficiency (Wettergren et al., 2012). Yang and colleagues (Yang and Hon, 2009) investigated 5-FU-loaded chitosan nanoparticles whether they could be used as effective drug delivery systems and suggested that chitosan-drug conjugates are expected to overcome the problematic side effects, which were induced by 5-FU.

Our study was carried out to evaluate the antiangiogenic efficacy of Chitosan nanoparticles as a drug carrier for 5-Fluorouracil on mice suffering from Ehrlich carcinoma.

\section{MATERIAL AND METHODS}

\section{Ehrlich ascites carcinoma cells (EACs)}

EACs were obtained from the Egyptian National Cancer Institute (NCI), Cairo University. The tumor line was maintained in female Swiss albino mice by intra-peritoneal inoculation of 2.5 million cells per mouse. The EACs were counted before intraperitoneal injection using the bright line hemocytometer and dilution were made by physiological sterile saline solution and desired numbers of cells were injected in a volume of $0.2 \mathrm{ml}$.

\section{Experimental animals}

Adult female Swiss albino mice of 8 weeks old and 22-25g weight purchased from the breeding unit of the Egyptian Organization for Biological Products and Vaccines were used in this study. The animals were maintained on a commercial standard pellet diet and tap water. All the experimental procedures were carried out according to the principles and guidelines of the Ethics Committee of the National Research Centre conformed to "Guide for the care and use of Laboratory Animals" for the use and welfare of experimental animals, published by the US National Institutes of Health (NIH publication No. 85-23, 1996).

\section{Preparation of nanoparticles}

Nanoparticles were prepared according to the method described by Banerjee et al. (2002). Briefly, the surfactant sodium bis (2-ethylhexyl) sulfosuccinate (AOT) was dissolved in n-hexane. $400 \mu$ of $0.1 \% \mathrm{w} / \mathrm{v}$ chitosan solution dissolved in acetic acid was added to $40 \mathrm{ml}$ of $0.04 \mathrm{M}$ AOT solution with continuous stirring at room temperature. The solvent was evaporated off in a rotary evaporator and dry the mass in $20 \mathrm{ml}$ of Tris- $\mathrm{HCl}$ buffer ( $\mathrm{pH} \mathrm{8.0)}$ by sonication. $4 \mathrm{ml}$ of $30 \% \mathrm{CaCl} 2$ solution were added to precipitate the surfactant as calcium salt of diethylhexyl-sulpho-succinate. The precipitate was pelleted by centrifugation at $6000 \mathrm{rpm}$ for $15 \mathrm{~min}$ at $4{ }^{\circ} \mathrm{C}$. The cake of Ca was dissolved in $10 \mathrm{ml} \mathrm{n}$-hexane and 
washed two to three times with $1 \mathrm{ml}$ of Tris- $\mathrm{HCl}$ buffer. The phase-separated aqueous layer was drained out and centrifuged. The total aqueous dispersion of nanoparticles was dialyzed and lyophilized.

Mitra et al. (2001) reverse micellar method was used to prepare 5FUCNPs. $200 \mu \mathrm{l}$ of 5-Flourouracil $(10 \mathrm{mg} / \mathrm{ml})$ was added after addition of chitosan solution in $6 \% \mathrm{v} / \mathrm{v}$ acetic acid. Size and morphology of nanoparticles were done by using transmission electron microscopy (TEM). Samples for TEM were prepared using the clear solution of nanoparticles. The sample solution was put on a formvar coated grid. On this grid, a drop of the sample solution (containing dispersed nanoparticles) was placed and allowed to air-dry. A TEM picture was taken by a JOEL JEM 2000 EX200 microscope.

\section{In vitro study}

Cytotoxicity effects of the nanoparticles on tumor cells were determined according to the method of El- Merzabani et al., (1979). In order to detect the cytotoxicity of 5FUCNPs, EACs were treated with nanoparticles at the concentrations of $1,2,3,4,5,6,7,8,9,10,20,30,40,50,60,70,80,90$ and $100 \mu \mathrm{g} / \mathrm{ml}$. The EACs were obtained by needle aspiration of ascitess fluid from the pre inoculated mice under aseptic condition using ultra violet laminar air flow system. The percentages of nonviable cells were determined by counting dead and viable EACs. To differentiate between dead and viable EAC cells, trypan blue stain was used. Then the percentages of nonviable cells (NVC) were calculated according to the following equations $\% \mathrm{NVC}=\mathrm{C} / \mathrm{T}$ $\mathrm{X} 100$, where (C) is number of nonviable cells and (T) is total number of viable cells.

\section{In vivo study}

To assess a solid mass of Ehrlich tumor, $0.2 \mathrm{ml}$ EAC cells containing $2.5 \times 10^{6}$ cells/mouse were inoculated subcutaneously in the back of the neck region of female mouse. After 7 days of tumor inoculation the experimental animals were subdivided into 2 groups having 10 animals in each group. Tgroup: Female Swiss albino mice bearing Ehrlich carcinoma without any treatment and 5FUCNPsgroup: Swiss albino mice bearing Ehrlich carcinoma that given 5-FUCNPs at a dose level $0.5 \mathrm{mg} / \mathrm{kg} /$ day for 15 days.

\section{Monitoring the tumor size}

Tumor size was monitored twice or thrice weekly throughout the experiment. The tumor size being measured regularly using Vernier calipers and represented in terms of tumor size. The tumor size was estimated using the following formula: Tumor size $\left(\mathrm{mm}^{3}\right)=4(\mathrm{~A} / 2)(\mathrm{B} / 2)^{2}=0.52 \mathrm{AB}^{2}$, where $\mathrm{A}$ is the major axis and $B$ is the minor axis (Ghoneum et al., 2008). The mean tumor size with the corresponding standard error was calculated in each experimental group.

\section{Sample preparation}

21 days after tumor inoculation (ATI) of final treatment of each group, experimental animals were sacrificed. Immediately Ehrlich tumor and liver tissues were excised and rinsed in saline. Small part from tumor and liver tissues were placed in $10 \%$ phosphate-buffered formalin to be used in histopathological and apoptotic and necrotic examinations. The rest of the Ehrlich tumor and liver tissues were homogenates in cold isotonic sucrose to be used for the estimation of the biochemical assessed parameters.

\section{Histopathological examination}

Paraffin slide sections from tumor and liver tissues were stained with Hematoxylin and eosin.

\section{Biochemical analysis}

All biochemical analysis was performed in Ehrlich tumor and liver homogenate. The level of malondialdehyde (MDA), the end-product of lipid peroxidation was measured according to the method of Yoshioka et al. (1979). Glutathione concentration 
(GSH) was determined by the method of Beulter $\boldsymbol{e t}$ al. (1963). Also, serum (TNF- $\alpha$, VEGF and PDGF) were assayed by the standard sandwich enzymelinked immune-sorbent assay (ELISA) technique used for Quantitative Detection of Mouse TNF alpha, VEGF and PDGF Concentrations in Serum.

\section{Statistical analysis}

The obtained data were expressed as mean \pm standard error (SE). All data were analyzed statistically using one-way analysis of variance (ANOVA) followed by Student's t-test. Statistical Package for Social Sciences (SPSS) for Windows version 12.0 software was used for this analysis.

\section{RESULTS}

Figure 1. Represents the morphology and size of 5FUCNPs. TEM observations revealed that Chi- tosan have a spherical shape. However, 5FUCNPs of cross-linking $(10 \%)$ appear as small aggregates. Ultrafine 5-FUCNPs, as shown by the TEM images, spherical shaped uniform solid dense structure and have nearly uniform particle size distribution, which is very important for drug delivery. Average particle size of 5 FUCNPs is $60 \mathrm{~nm} \pm 19 \mathrm{~nm}$ and the size for distribution ranged from 40 to $100 \mathrm{~nm}$.

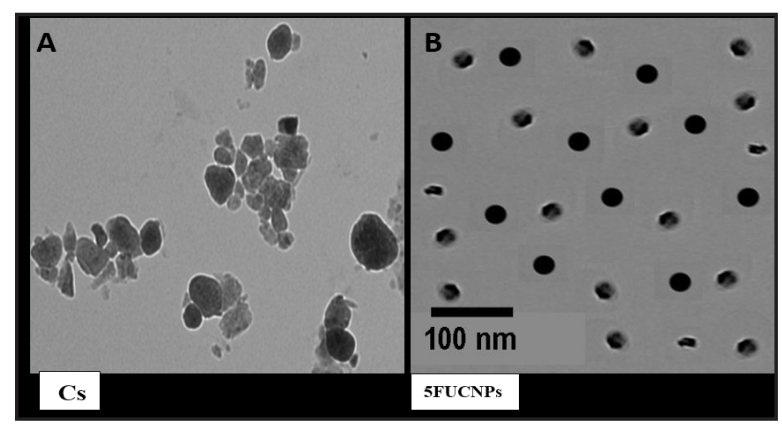

Fig. (1): Morphology and size of Nanoparticles.

Table (1): The effect of 5-Fluorouracil Chitosan nanoparticles (5FUCNPS) on the viability of Ehrlich asci tess carcinoma cells.

\begin{tabular}{|c|c|c|c|}
\hline \multicolumn{3}{|c|}{ 5-Florouracil - loaded Chitosan Nanoparticles (5FUCNPs) } \\
\hline $\begin{array}{c}\text { Nanoparticles } \\
\text { concentration } \\
(\boldsymbol{\mu g} / \mathbf{m L})\end{array}$ & \% of viable cells & \% of dead cells & \% of rupture dead cells \\
\hline 0 & 99 & 1 & - \\
\hline 1 & 99 & 1 & - \\
\hline 2 & 99 & 1 & - \\
\hline 3 & 99 & 1 & - \\
\hline 4 & 90 & 10 & - \\
\hline 5 & 90 & 10 & - \\
\hline 6 & 90 & 10 & 50 \\
\hline 7 & 70 & 30 & 20 \\
\hline 8 & 50 & 50 & 80 \\
\hline 9 & 40 & 60 & 100 \\
\hline 10 & 30 & 70 & 100 \\
\hline 20 & 10 & 90 & 100 \\
\hline 30 & 0 & 100 & 100 \\
\hline 40 & 0 & 100 & 100 \\
\hline 50 & 0 & 100 & 100 \\
\hline 60 & 0 & 100 & 100 \\
\hline 70 & 0 & 100 & - \\
\hline 80 & 0 & 100 & - \\
\hline 90 & 0 & 100 & - \\
\hline & & & \\
\hline & & & \\
\hline
\end{tabular}




\section{In vitro studies}

\section{Chemosensitivity of nanoparticles on Ehrlich ascites carcinoma:}

The cytotoxic effect of different concentrations of 5FUCNPs - on Ehrlich cells viability was evaluated in Table 1 and Figure 2. The low concentration $(10 \mu \mathrm{g} / \mathrm{ml})$ of 5 FUCNPs decreases the tumor cells viability by $70 \%$. The cytotoxicity of nanoparticle not only led to the death of Ehrlich carcinoma cells, but also led to burst of these dead cells at certain doses. The median lethal concentration of 5FUCNPs was $20 \mu \mathrm{g} / \mathrm{ml}$ leading to the death of $90 \%$ Ehrlich carcinoma cells.

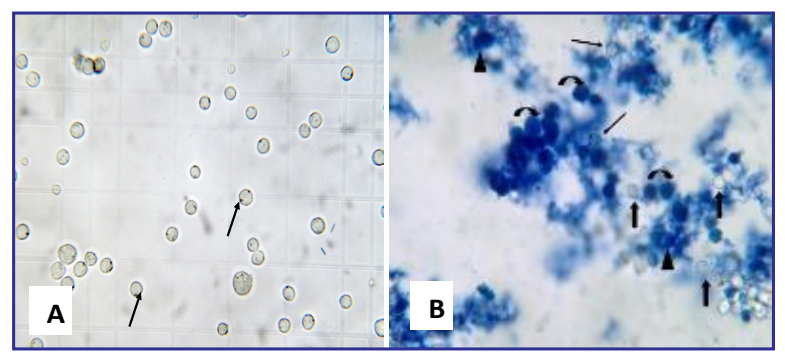

Fig. (2): Effect of 5FUCNPs on the viability of Ehrlich ascites carcinoma cell line. (A): Photomicrograph of Ehrlich carcinoma cells line representing $100 \%$ of viable cells $(\uparrow)$. (B) Photomicrograph of Ehrlich carcinoma cells line as affected by 5FUCNPs nanoparticles. Notice: viable ascites carcinoma cells ( ), underwent apoptotic ascites carcinoma cells $(\uparrow)$, rupture ascites carcinoma cells $(\boldsymbol{\Delta})$ and dead cells (curved arrow).

\section{Monitoring of Ehrlich carcinoma tumor size:}

Ehrlich tumor size is represented in Figure 3 it is clear that the inoculation of 2.5 million of Ehrlich ascitess carcinoma (EAC) cells in $2 \mathrm{ml}$ physiological saline in the back of the neck region of healthy normal mice produced a solid tumor with a mean size of $175 \pm 3.4 \mathrm{~mm}^{3}$ on the $7^{\text {th }}$ day after tumor inoculation (ATI). EC size exceeds $300 \mathrm{~mm}^{3}$ on the $9^{\text {th }}$ day ATI. The increase of EC size proceeds by days reaching $1733 \pm 2.0 \mathrm{~mm}^{3}$ on the $21^{\text {st }}$ day ATI.

When mice bearing Ehrlich carcinoma received by gavages $0.5 \mathrm{mg} / \mathrm{kg}$ /day 5FUCNPs for 15 days beginning from the $7^{\text {th }}$ day ATI, a significant reduction in tumor size was recorded $194 \pm 1.4 \mathrm{~mm}^{3}$ on the $9^{\text {th }}$ day ATI and reaching $459 \pm 1.5 \mathrm{~mm}^{3}$ on the $21^{\text {st }}$ day ATI.

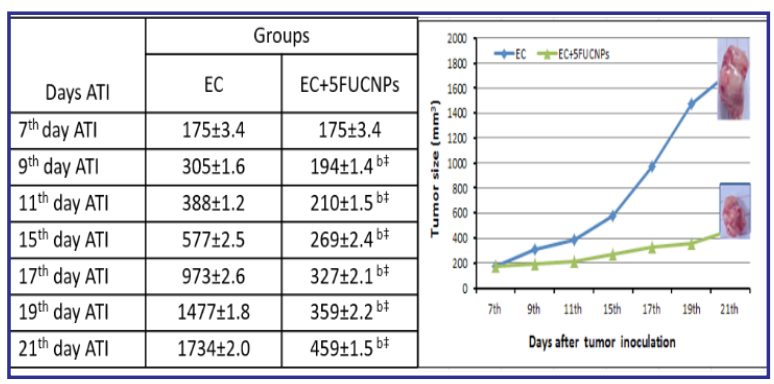

Fig. (3): Effect of 5FUCNPs on Ehrlich Carcinoma (EC) tumor size.

Table (2) : Effect of 5FUCNPs on TNF- $\alpha, P D G F$ and VEGF levels $(\mathrm{pg} / \mathrm{ml})$ of mice bearing Ehrlich carcinoma.

\begin{tabular}{|c|c|c|c|}
\hline \multirow{2}{*}{ Parameter } & Groups & EC & E.5FUCNPs \\
\hline \multirow{3}{*}{ TNF- $\alpha$} & Mean \pm SE & $117.5 \pm 2.7$ & $61.6 \pm 1.8$ \\
\cline { 2 - 4 } & \% of change from EC & 0 & -47.5 \\
\hline \multirow{3}{*}{ PDGF } & Mean \pm SE & $138.5 \pm 5.0$ & $80.3 \pm 2.7$ \\
\cline { 2 - 4 } & \%of change from EC & 0 & -41.8 \\
\hline \multirow{3}{*}{ VEGF } & Mean \pm SE & $207.9 \pm 7.7$ & $83.9 \pm 2.4$ \\
\cline { 2 - 4 } & \%of change from EC & 0 & -59.6 \\
\hline
\end{tabular}

Values are expressed as Means of 6 records \pm standard Error $(M \pm S E)$

bł: very highly significant against EC at $(P \leq 0.001)$. 


\section{Oxidative stress markers level in tumor and liver tissues of tumor bearing mice}

As shown in in Table 3, treatment of the experimental mice-bearing EC with 5FUCNPs produced a significant increase in tumor MDA by $29 \%$ and significant decrease in tumor GSH by $-42.4 \%$ compared with EC group. Meanwhile, treatment of the experimental mice-bearing EC with 5FUCNPs produced a significant decrease in liver MDA by -16.9 and a high significant increase in liver GSH level by $77.2 \%$ against $\mathrm{EC}$ group.

Table (3) : Effect of 5FUCNPs on TNF- $\alpha, P D G F$ and VEGF levels ( $\mathrm{pg} / \mathrm{ml})$ of mice bearing Ehrlich carcinoma.

\begin{tabular}{|c|c|c|c|c|c|}
\hline \multicolumn{2}{|c|}{ Groups } & \multicolumn{2}{|r|}{ EC } & \multicolumn{2}{c|}{ E.5FUCNPs } \\
\cline { 3 - 6 } Parameters & Tumor & Liver & tumor & Liver \\
\hline $\begin{array}{c}\text { MDA } \\
(\mu \text { mol/gm } \\
\text { tissue })\end{array}$ & Mean \pm SE & $222.6 \pm 2.2$ & $109.8 \pm 1.3$ & $141.6 \pm 1.9 \mathrm{~b}^{\ddagger}$ & $185 \pm 1.5 \mathrm{~b}^{\star}$ \\
\cline { 2 - 6 } & \% of change from EC & 0 & 0 & 29 & -16.9 \\
\hline $\begin{array}{c}\text { GSH } \\
\text { (mg } / \text { gm fresh } \\
\text { tissue })\end{array}$ & Mean \pm SE & $2.2 \pm 0.09$ & $3.3 \pm 0.06$ & $1.9 \pm 0.04 \mathrm{~b}^{*}$ & $3.9 \pm 0.09$ \\
\cline { 2 - 6 } & \%of change from EC & 0 & 0 & -42.4 & 77.2 \\
\hline
\end{tabular}

Values are expressed as Means of 6 records \pm standard Error $(M \pm S E)$

bł: very highly significant against EC at $(P \leq 0.001)$.

\section{Histopathological examination of the Ehrlich carcinoma:}

Histopathological examination of the Ehrlich carcinoma tumor under light microscope showed compactness and aggregation of the tumor tissue cells spread subcutaneously within the soft tissues in the neck region. Ehrlich carcinoma tumor showed groups of large, round and polygonal cells, with pleomorphic shapes, hyperchromatic nuclei and binucleation. Several degrees of cellular and nuclear pleomorphism were seen (Figure 4 A). Treatment of female mice bearing Ehrlich carcinoma tumor by 5 FUCNPs recorded great destruction of tumor tissue represented by the appearance of dead (arc) and necrotic cells (star) (Figure 4B).

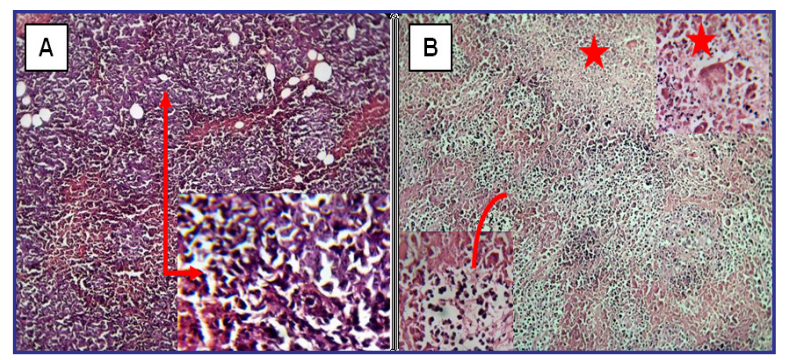

Fig. (4): Photomicrograph represents control Ehrlich carcinoma in mice (A), treated by 5FUCNPs (B). (H \& E x 100).

\section{Histopathological examination of the liver tissue in different animal's groups:}

The liver sections of mice bearing Ehrlich carcinoma showed accumulation of Ehrlich carcinoma cells (ECs) around congested portal blood vessels with completely haemolysed red blood cells (RBCs) in the portal vein (Figure 5A). while treatment of female mice with $0.5 \mathrm{mg} / \mathrm{kg} /$ day 5 FUCNPs showed aggregation of inflammatory cells around the hepatic portal and hepatic veins with no appearance of tumor cells metastasis (Figure 5B).

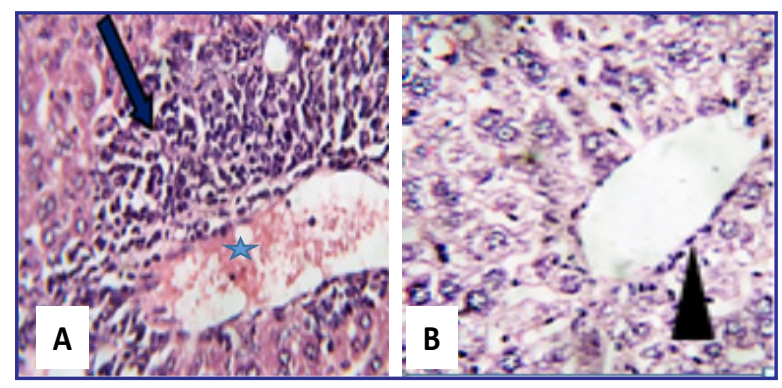

Fig. (5): Photographs of sections in liver of mice bearing Ehrlich carcinoma. A: Liver tissue of mice bearing Ehrlich carcinoma represented accumulation of ECs (blocked arrow) around a congested portal blood vessel (star) with completely haemolysed RBCs. B: Liver tissue of mice treated with 5FUCNPs represents some appearance of less metastatic ECs ( $\Delta$ ). (H\&E stain X 400). 


\section{DISCUSSION}

Conventional chemotherapeutic drugs are distributed nonspecifically in the body where they affect both cancerous and healthy cells, resulting in dose-related side effects and inadequate drug concentrations reaching the tumor. Recent progress in cancer nanotechnology raises exciting opportunities for specific drug delivery. Nanoparticles, particularly in the size range from $10 \mathrm{~nm}$ to $100 \mathrm{~nm}$, are emerging as a class of therapeutics for cancer treatment (Wang et al., 2009). Chitosan is an interesting natural material occurring in abundance in the environment. Its excellent biocompatibility and several advantages due to its unique polymer cationic character render it highly useful for pharmaceutical applications (Thanoo et al., 1992). Chitosan nanoparticles (CNPs) have been previously synthesized as drug delivery for 5-FU. In the present study the cytotoxicity of 5FUCNPs on Ehrlich carcinoma cell line was carried out. The present study demonstrated that 5FUCNPs could exert a very high cytotoxicity against Ehrlich ascitess carcinoma cell line. The median lethal concentration of 5FUCNPs was $20 \mu \mathrm{g} / \mathrm{ml}$ and at concentration of $30 \mu \mathrm{g} / \mathrm{ml} 5$ FUCNPs all Ehrlich carcinoma cells appeared to be burst. The cytotoxicity effect of nanoparticles is due to their adherence to the cell membrane, particle internalization and degradation of products in the cell culture medium or inside the cells (Abbasalipourkabir et al., 2011). Chitosan nanoparticles are positively charged due to the cationic characteristics of chitosan ( $\mathrm{Hu}$ et al., 2002). 5FUCNPs could be first adsorbed onto the negatively charged tumor cell membrane by electron interaction, then exhibit antitumor effects by damaging membrane and disrupting organelle, and finally lead to cell death with the structure breakdown (Qi et al., 2005). Mitra et al. (2001) studied the in vivo efficacy of using chitosan nanoparticles as a drug carrier and suggest that encapsulation of the conjugate in nanoparticles not only reduces the side effects, but also improves its therapeutic efficacy in the treatment of solid tumors. In the present study when experimental animal's gavage with 5FUCNPs, a delay in tumor size was recorded (El-Merzabani et al., 1979). The mechanism of nanoparticles in reducing the tumor size may be through the long-circulating nanoparticulate carriers. They can efficiently deliver the 5-FU to solid tumors by exploiting the enhanced permeability and retention (EPR) effect and thus can significantly enhance the therapeutic index of 5-FU or improve reducing undesirable side effects. Studies recorded that ultra-low size particles made of hydrogel polymer such as chitosan can efficiently be targeted to the tumor tissue through the combined effects of extravasation and long circulation in blood (Savita and Amarnath, 2009). On the other hand, context with the findings of many studies with the role of 5FUCNPs in tumor destruction, treatment of the experimental animals with 5FUCNPs great destruction and appearance of dead and necrotic cells represents many zones of sporadic underwent apoptotic cells in addition to the presence of apoptotic zone were detected in Ehrlich carcinoma (Zhang et al., 2008). There are many evidence showing that nanoparticles increase ROS production and in different types of cancer cells (Peters et al., 2007). In the present study treatment of experimental animals bearing Ehrlich carcinoma by 5FUCNPs recorded a significant increase in tumor MDA level compared to untreated tumor bearing group. The inverse linear relationship between the ROS level and the GSH level indicated that free radical species were generated by exposure to nanoparticles which reduced intracellular antioxidant levels (Fen et al., 2009). Treatment of experimental animals bearing Ehrlich carcinoma by 5 FUCNPs developed a significant decrease in tumor GSH level compared to the level in tumor tissue of mice bearing Ehrlich carcinoma group. Furthermore, it has been well documented that GSH depletion cause mitochondrial dysfunction and changes in expression of distinct genes and pathways related to inflammatory responses and apoptosis including MAPK/ERK kinase, NFjB, MIP-2, caspase- 3, Bcl2 (Driscoll, 2000; Fubini and Hubbard, 2003). 
It has been demonstrated in many in- vivo studies that angiogenesis is responsible for tumor growth and metastasis. VEGF, PDGF are angiogenic factors that play an important role in the process of tumor angiogenesis and in turn in tumor progression and metastasis (Dor et al., 2001). The present study shows that the treatment of the experimental micebearing EC with 5FUCNPs produced a significant decrease in the levels of TNF- $\alpha$, PDGF and VEGF, against Ehrlich Carcinoma-bearing group due to the synergetic antiangiogenic effect of 5-FU together with CNPs which normalizes tumor vasculature, facilitates improved chemotherapy delivery, and prevents the recruitment of progenitor cells from the bone marrow (Kerbel, 2008). TNF- $\alpha$ was the major mediator of inflammatory response (Feghali and Wright, 1997). In EC bearing mice, the elevated cytokine (TNF- $\alpha$ ) level may be attributed to oxidative stress. TNF- $\alpha$ act as tumor promoter because it stimulates cancer cells' growth, proliferation, invasion and metastasis through activation of NF- $\kappa B$ signaling pathway (Ahmed et al., 2001). Our study showed reduced TNF- $\alpha$ level in the sera of 5FUCNPs treated mice. The decrease in TNF- $\alpha$ levels suggests an anti-inflammatory effect which might delay cancer progression (Agrawal et al., 2011).

Furthermore, the present study showed that the experimental mice bearing- EC recorded a significant increase in the liver LPO and a high significant decrease in GSH levels as a result of increased oxidative stress in liver tissue. The oxidative stress in the liver of mice bearing-EC induced hepatocyte cell death by either apoptosis or necrosis leading to liver injury and loss of liver function (Okuda $\boldsymbol{e t}$ al., 2002). While treatment of experimental animals bearing Ehrlich carcinoma with 5FUCNPs recorded a significant decrease in tumor MDA and a high significant increase in GSH levels compared to untreated tumor bearing group These previous findings gave the fact that chitosan nanoparticles in combined with 5-FU diminished the damaged effect of free radicals, hence decreasing the level of lipid peroxidation resulting in decreasing liver oxidative stress and normalization of the liver tissue with histopathological disappearance of metastatic Ehrlich cells from liver tissue.

\section{CONCLUSION}

CNPs plays an important role in drug delivery of 5-FU through enhancement its cytotoxic, anti- tumor effect and diminishing its side effect. in addition, CNPs together with 5-FU inhibit Ehrlich Carcinoma growth by inhibiting the production of new tumor vacuolization

\section{REFERENCES}

- Abbasalipourkabir, R.; Salehzadeh, A. and Abdullah, R. (2011): Cytotoxicity Effect of Solid Lipid Nanoparticles on Human Breast Cancer Cell Lines. Biotechnology;10: 528.

- Agrawal, S.S.; Saraswati, S.; Mathur, R. and Pandey, M. (2011): Cytotoxic and antitumor effects of brucineon Ehrlich ascitess tumour and human cancer cell line. Life Sci.; 89: 147.

- Ahmed, M.I.; Salahy, E.E.; Fayed, S.T.; El-Hefnawy, N.G. and Khalifa, A. (2001): Human papillomavirus infection among Egyptian females with cervical carcinoma: relationship to spontaneous apoptosis and TNFalpha. Clin. Biochem.; 34(6): 491.

- Alvarez-Lorenzo, C.; Sosnik, A. and Concheiro, A. (2011): PEO-PPO block copolymers for passive micellar targeting and overcoming multidrug resistance in cancer therapy. Curr. Drug Targets; 12: 1112.

- Banerjee T.; Mitra, S. Singh, AK.; Sharma RK. and Maitra, AN. (2002): Preparation characterization and biodistribution of ultrafine chitosan nanoparticles. Int. J. Pharm.; 243(1-2): 93.

- Beulter, E.; Duron, O. and Kelly, BM. (1963): Improved method for the determination of blood glutathione. J. Lab. Clin. Med.; 61: 882.

- Burns, E.R. and Beland, S.S. (1983): Induction 
by 5-fluorouracil of major phase difference in the circadian profiles of DNA synthesis between the Ehrlich ascitess carcinoma and five normal organs. Cancer Lett.;20: 235.

- Dor, Y.; Porat, R. and Keshet, E. (2001): Vascular endothelial growth factor and vascular adjustments to perturbations in oxygen homeostasis. Am. J. Physiol. Cell Physiol.; 280:1367.

- Driscoll, KE. (2000): TNFa and MIP-2: role in particle-induced inflammation and regulation by oxidative stress. Toxicol. Lett.; 112-113: 177.

- El-Merzabani.; MM., El-Aaser, AA.; Attia, MA.; El- Duweini, AK. and Ghazal, AM. (1979): Screening system for Egyptian plants with potential anti-tumour activity. Planta Med.; 36: 150.

- Estanqueiro, M.; Amaral, M.H.; Conceic, J. and Lobo, J.M.S. (2015): Nanotechnological carriers for cancer chemotherapy: the state of the art. Colloids Surf. B;126: 631 .

- Feghali, CA. and Wright, TM. (1997): Cytokines in acute and chronic inflammation. Front Biosci.; 2: d12.

- $\quad$ Fen, W.; Feng, G.; Minbo, L.; Huihui, Y.; Yongping, H. and Jianwen, L. (2009): Oxidative stress contributes to silica nanoparticle-induced cytotoxicity in human embryonic kidney cells. Toxicol. In Vitro; 23: 808 .

- Fubini, B. and Hubbard, A. (2003): Reactive oxygen species and reactive nitrogen species generation by silica in inflammation and fibrosis. Free Radic. Biol. Med.; 34: 1507.

- Ghoneum, M.; Badr El-Din, NK.; Noaman, E. and Tolentino, L. (2008): Saccharomyces cerevisiae, the Baker's Yeast, suppresses the growth of Ehrlich carcinoma-bearing mice. Cancer Immunol. Immunother.; 57: 581 .

- Hu, Y.; Jiang, X.; Ding, Y.; Ge, H.; Yuan, Y. and Changzheng, Y. (2002): Synthesis and characterization of chitosan-poly (acrylic acid) nanoparticles. Biomaterials; 23: 3193.
- Kerbel, R.S. (2008): Tumor angiogenesis. The New England J. of Med.; 358(19): 2039.

- Mitra S.; Gaur, U.; Ghosh, P.C., and Maitr, A.N. (2001): Tumor targeted delivery of encapsulated dextran-doxorubicin conjugate using chitosan nanoparticles as carrier. J. Control. Release; 74(1-3): 317.

- Okuda, M.; Li, K.; Beard, M. R.; Showalter, L. A.; Scholle, F.; Lemon, S. M. and Weinman, S. A. (2002): Mitochondrial injury, oxidative stress, and antioxidant gene expression are induced by hepatitis $\mathrm{C}$ virus core protein. Gastroenterol.;122(2): 366.

- Ozaslan, M.; Karagoz, I.D.; Kilic, I.H. and Guldur, M.E. (2011): Ehrlich ascitess carcinoma. Afr. J. Biotechnol.; 10(13): 2375.

- $\quad$ Peters, K.; Unger, RE.; Gatti, AM.; Sabbioni, E.; Tsaryk, R. and Kirkpatrick, CJ. (2007): Metallic nanoparticles exhibit paradoxical effects on oxidative stress and proinflammatory response in endothelial cells in vitro. Int. J. Immunopathol. Pharmacol.; 20: 685.

- Qi, L.; Xu, Z.; Li, Y.; Jiang, X. and Han, X. (2005): In vitro effects of chitosan nanoparticles on proliferation of human gastric carcinoma cell line MGC803 cells. World J. Gastroenterol.; 11(33): 5136.

- Ravi Kumar, M.N.V. (2000): A review of chitin and chitosan applications. React. Funct. Polym.; 46: 1.

- Savita, B. and Amarnath, M. (2009): Dextrandoxorubicin/chitosan nanoparticles for solid tumor therapy. Wiley Interdiscip. Rev. Nanomed. Nanobiotechnol.; 1(4): 415.

- Thanoo, B.C.; Sunny, M.C. and Jayakrishnan, A. (1992): Cross-linked chitosan microspheres: preparation and evaluation as a matrix for the controlled release of pharmaceuticals. J. Pharm. Pharmacol.; 44: 283.

- Wang, X..; Wang, Y.; Chen, Z. and Shin, D. (2009): Advances of Cancer Therapy by Nanotechnology. Cancer Res. Treat.; 41(1):1. 
- Wettergren, Y.; Carlsson, G.; Odin, E. and Gustavsson, B. (2012): Pretherapeutic uracil and dihydrouracil levels of colorectal cancer patients are associated with sex and toxic side effects during adjuvant 5-fluorouracil-based chemotherapy. Cancer; 118: 2935.

- Yang, H.C. and Hon, M.H. (2009): The effect of the molecular weight of chitosan nanoparticles and its application on drug delivery. Microchem. J.; 92(1): 87.

- Yoshioka, T.; Kawada, K.; Shimada, T. and Movi M. (1979): Lipid per oxidation in maternal and cord blood and protective mechanism against activated oxygen toxicity in the blood. Am. J. Obstet. Gynecol.; 135: 372 .
- Zhang, D.Y.; Shen, X.Z.; Wang, J.Y.; Dong, L.; Zheng, Y.L. and Wu, L.L. (2008): Preparation of chitosan- poly aspartic acid-5 fluorouracil nanoparticles and its anti-carcinoma effect on tumor growth in nude mice. World J. Gastroenterol.; 14: 3554. 